\title{
Functional Status Before and During Acute Hospitalization and Readmission Risk Identification
}

\author{
Orly Tonkikh, MA ${ }^{1 *}$, Efrat Shadmi, $\mathrm{PhD}^{1,2}$, Natalie Flaks-Manov, MPH${ }^{2}$, Moshe Hoshen, $\mathrm{PhD}^{2}$, Ran D. Balicer, PhD ${ }^{2,3}$, \\ Anna Zisberg, $\mathrm{PhD}^{1}$
}

\begin{abstract}
${ }^{1}$ The Cheryl Spencer Department of Nursing, Faculty of Social Welfare and Health Sciences, University of Haifa, Haifa, Israel; ${ }^{2}$ Clalit Research Institute, Chief Physician's Office, Clalit Health Services, Tel-Aviv, Israel; ${ }^{3} E$ pidemiology Department, Faculty of Health Sciences, Ben-Gurion University of the Negev, Beer-Sheva, Israel.
\end{abstract}

BACKGROUND: Recent efforts to prevent readmissions are increasingly focusing on early identification of high-risk patients.

OBJECTIVE: To test whether information on functioning during hospitalization contributes to the ability to accurately identify older adults at high risk of readmission beyond their baseline risk.

DESIGN: Prospective cohort study.

SETTING: Internal medicine wards at 2 medical centers.

PATIENTS: Five hundred fifty-nine community-dwelling older adults (aged $\geq 70$ years) discharged to their homes.

MEASUREMENTS: Data on unplanned 30-day readmissions were retrieved from electronic health records. Data on at-admission activities of daily living (ADL) and in-hospital ADL decline were collected using validated questionnaires. Multivariate logistic regression was used to model the association between functioning and readmission controlling for known risk factors.
RESULTS: Higher in-hospital ADL decline was significantly associated with readmission (odds ratio for each 10-point decrease in $A D L=1.32,95 \%$ confidence interval $=1.02-$ 1.72) but did not contribute to the overall discrimination of the model, as compared with the at-admission data (C statistic $=0.81$ for each model). Identifying high-risk (10th highest percentile) patients by the at-admission model did not detect $7 / 55(12.7 \%)$ of patients who would have been categorized as high risk if risk identification was postponed to the discharge date and included data on in-hospital ADL decline.

CONCLUSIONS: The study highlights the ability to identify patients at high risk for readmission already early in the index hospitalization using data on functioning, nutrition, chronic morbidity, and prior hospitalizations. Nonetheless, at-discharge functional assessment can detect additional patients whose readmission risk changes during the index hospitalization. Journal of Hospital Medicine 2016;11:636641. (C) 2016 Society of Hospital Medicine
A continuing focus on readmission prevention as a means to improve quality and reduce waste and costs has led to abundant research on the identification of factors that put older patients at risk for readmission. ${ }^{1,2}$ Recently, research has focused on the development of prediction tools that are based on information from electronic health records (EHR) and that enable early, atadmission, identification of patients at high risk for readmission. ${ }^{3-5}$ Through such identification, patients are already targeted for inclusion in readmission-prevention interventions early in their hospital stay. ${ }^{6,7}$ Yet, the ability to rely on these early-identification tools is contingent on the stability of risk during the hospital stay. Of the inhospital factors that can affect readmission, change in functioning has been identified as a potentially major contributor, especially in older patients. ${ }^{8}$

*Address for correspondence and reprint requests: Orly Tonkikh, The Cheryl Spencer Department of Nursing, Faculty of Social Welfare and Health Sciences, University of Haifa, Haifa 31905, Israel; Telephone: 972-508885845; Fax: 972-48288017; E-mail: orly.tonkikh@gmail.com

Additional Supporting Information may be found in the online version of this article.

Received: November 17, 2015; Revised: March 30, 2016; Accepted: April 5, 2016

2016 Society of Hospital Medicine DOI 10.1002/jhm.2595

Published online in Wiley Online Library (Wileyonlinelibrary.com).
Older adults' deterioration in functioning is a common, troubling phenomenon. Approximately $20 \%$ of patients older than 70 years and hospitalized for a medical illness deteriorate in functioning during the hospitalization. ${ }^{9}$ Recent evidence points to the contribution of functioning to 30-day readmission risk, with studies showing that pre- or posthospitalization functional impairment is associated with a 1.5 to 3 times greater likelihood of readmission. ${ }^{10-13}$ Whether in-hospital changes in functioning add to patients' baseline risk, however, is unknown. The only readmission study that examined functional decline during hospitalization was a retrospective study performed in a population of older adults receiving rehabilitation services and therefore may not be generalizable to acutely hospitalized adults. ${ }^{14}$ To fill the current knowledge gap, we examined whether in-hospital functional decline significantly contributes to at-admission readmission risk factors in its ability to accurately identify high readmission risk in older adults hospitalized in internal medicine units.

\section{METHODS}

Design and Participants

Previously collected data from a prospective cohort study, Hospitalization Process Effects on Functional Outcomes and Recovery (HoPE-FOR), designed to 
assess the effect of hospitalization-care processes on functional outcomes in older adults, were combined with EHR data. The population considered for recruitment to HoPE-FOR comprised older patients (aged $\geq 70$ years) admitted during the period 2009 to 2011 to 1 of the 8 internal medicine wards at 2 tertiary medical centers in Israel. Patients recruited for the study had an unplanned admission, and were not completely dependent in their basic functions. Cognitively impaired patients (scoring 5 or less on the Short Portable Mental Status Questionnaire [SPMSQ] ${ }^{15}$ ) who had no available caregiver and patients admitted for stroke, coma, or respiratory failure requiring mechanical ventilation were not eligible for participation in the study. The recruitment process for the study is fully described elsewhere. ${ }^{16,17}$

At-admission functional, cognitive, mental, and nutritional status were assessed during the first 48 hours of hospitalization. Additional functional assessment was performed at discharge. Data on severity of acute disease, length of stay (LOS), and mortality were collected from the hospitals' EHR. Preadmission healthcare utilization and readmission information were retrieved from the EHR database of Clalit Health Services (Clalit), a large not-for-profit integrated healthcare provider and insurer in Israel; more than $80 \%$ of the HoPE-FOR population were Clalit members.

Of the 969 community-dwelling participants recruited to the HoPE-FOR study, a subset of 758 $(78 \%)$ members of Clalit was used for the current study, as data on readmissions to any general hospital were accurately and readily available from Clalit's EHR system. ${ }^{18}$ Of those, we excluded 199 due to the following reasons: $13(2 \%)$ died during the hospitalization, $46(6 \%)$ transferred to another ward, $16(2 \%)$ were discharged to a post-acute care facility, and 124 (16\%) dropped-out from the HoPE-FOR study during the hospitalization (due to unavailability because of intensive tests or procedures ${ }^{16}$ ) or had missing data on the main variables, leaving a final sample of 559 participants. The admission functional, cognitive, and clinical status; LOS; and readmission rates of the participants who dropped-out were comparable to those of participants retained in the final sample except for albumin levels and age (see Supporting Information, Appendix A, in the online version of this article). The study was approved by the institutional reviews boards of each of the hospitals, Clalit, and the Israeli Ministry of Health.

\section{Variables and Instruments \\ Outcome Measure}

Readmission was defined as any unplanned hospitalization at any of 27 general hospitals in Israel, occurring within 30 days of discharge from the index hospitalization.

\section{Predictors}

We collected information on baseline and in-hospital characteristics. Baseline characteristics included chronic clinical conditions known as risk factors for readmission; number of different drugs as classified by the fifth level of the Anatomical Therapeutic Chemical classification system; number of hospitalizations in the year preceding the index hospitalization; clinical, functional, cognitive, mental, and nutritional status at admission; and sociodemographic characteristics.

Chronic conditions included: congestive heart failure (HF), chronic renal failure (CRF), chronic obstructive pulmonary disease (COPD), diabetes mellitus, ischemic heart disease, arrhythmia, malignancy, and asthma, and were retrieved from the Clalit's EHR data warehouse. ${ }^{19}$ Severity of acute disease was assessed with the Acute Physiology and Chronic Health Evaluation. ${ }^{20}$ Functional status was measured as self-reported independence in performing basic activities of daily living $(\mathrm{ADL}),{ }^{21}$ using the modified Barthel Index (mBI). ${ }^{22}$ The $\mathrm{mBI}$ consists of 10 items including personal hygiene, bathing, eating, toileting, dressing, chair/bed transfers, ambulation, stair climbing, and bowel and bladder control. Each item is ranked on 5-point scale, indicating the amount of assistance required in functional independence in each task. The scores are summarized into a total score ranging from 0 (totally dependent) to 100 (fully independent).

Cognitive status was assessed using the SPMSQ. ${ }^{15}$ Mental status was assessed based on self-report of depressive and anxiety symptoms using the 10-item Tucker's short Zung Instrument (TSZI) ${ }^{23}$ and the 10item Anxiety Symptoms Questionnaire Short Anxiety Screening Test (SAST), ${ }^{24}$ respectively. Both the TSZI and the SAST were validated in Hebrew as screening tools in older adults. ${ }^{24,25}$

Assessment of nutritional status included malnutrition risk (Malnutrition Universal Screening Tool [MUST]) and admission serum albumin level (g/dL). MUST provides classification into 3 malnutrition risk groups: low (being overweight or obese, with no loss of weight or loss of less than $5 \%$ of the weight and without expectation of fasting), medium (normal body mass index [BMI] or weight loss of 5-10\%), and high (normal BMI and inadequate weight loss or malnutrition by $\mathrm{BMI}$ and/or $10 \%$ weight loss and/or expectations of fasting). ${ }^{26}$ Classification of BMI in the current study was according to thresholds for older adults. ${ }^{27}$

In-hospital risk factors include LOS, a well-known risk factor of readmission ${ }^{28}$ and ADL decline during the index hospitalization. ADL decline was defined as the change in $\mathrm{mBI}$ score, calculated by subtracting the discharge score from the at-admission score and transforming negative scores (functional improvement) to 0 .

\section{Statistical Analysis}

The relationship between each of the study variables and readmission was examined using $\chi^{2}$ tests for 


\begin{tabular}{|c|c|c|c|c|}
\hline Characteristic & Entire Cohort, N = 559 & No Readmission, $\mathrm{n}=474$ & 30-Day Readmission, $\mathrm{n}=85$ & $P$ Value \\
\hline \multicolumn{5}{|l|}{ Baseline characteristics } \\
\hline \multicolumn{5}{|l|}{ Sociodemographic characteristics } \\
\hline Age, y, mean $\pm S D$ & $78.8 \pm 5.6$ & $78.7 \pm 5.6$ & $79.7 \pm 6.6$ & 0.19 \\
\hline Male, $n(\%)$ & $274(49.0)$ & $222(46.8)$ & $52(61.2)$ & 0.015 \\
\hline Living alone, $\mathrm{n}(\%)$ & $167(29.9)$ & $148(31.2)$ & $19(22.4)$ & 0.10 \\
\hline Education, y, mean \pm SD & $9.6 \pm 5.0$ & $9.8 \pm 4.9$ & $8.7 \pm 5.3$ & 0.074 \\
\hline \multicolumn{5}{|l|}{ Chronic condition, n (\%) } \\
\hline Congestive heart failure & $169(30.2)$ & $130(27.4)$ & $39(45.9)$ & 0.001 \\
\hline Chronic renal failure & $188(33.6)$ & $138(29.1)$ & $50(58.8)$ & $<0.001$ \\
\hline Chronic obstructive pulmonary disease & $93(16.6)$ & $77(16.2)$ & $16(18.8)$ & 0.56 \\
\hline Diabetes mellitus & $249(44.5)$ & $212(44.7)$ & $37(43.5)$ & 0.84 \\
\hline Ischemic heart disease & $353(63.1)$ & $295(62.2)$ & $58(68.2)$ & 0.29 \\
\hline Arrhythmia & $242(43.3)$ & $192(40.5)$ & $50(58.8)$ & 0.002 \\
\hline Malignancy & $176(31.5)$ & $132(27.8)$ & $44(51.8)$ & $<0.001$ \\
\hline Asthma & $72(12.9)$ & $61(12.9)$ & $11(12.9)$ & 0.99 \\
\hline No. of medications prescribed year before index hospitalization, mean \pm SD & $12.1 \pm 5.7$ & $11.9 \pm 5.5$ & $13.7 \pm 6.3$ & 0.007 \\
\hline \multicolumn{5}{|l|}{ Prior hospitalizations } \\
\hline №. of hospitalizations the year before index hospitalization, mean $\pm S D$ & $1.2 \pm 1.6$ & $1.00 \pm 1.3$ & $2.20 \pm 2.2$ & $<0.001$ \\
\hline \multicolumn{5}{|l|}{ At-admission health status } \\
\hline APACHE $\|(0-71)$, mean \pm SD & $11.5 \pm 4.4$ & $11.2 \pm 4.2$ & $12.9 \pm 4.6$ & 0.003 \\
\hline $\mathrm{ADL}(\mathrm{mBI})(0-100)$, mean \pm SD & $76.9 \pm 28.9$ & $78.4 \pm 28.4$ & $68.7 \pm 30.4$ & 0.004 \\
\hline Cognitive impairment (SPMSQ $\leq 5), n(\%)$ & $8.1 \pm 2.2$ & $8.1 \pm 2.2$ & $7.9 \pm 2.2$ & 0.32 \\
\hline Depression symptoms (TZI $\geq 70), \mathrm{n}(\%)$ & $106(19.0)$ & $89(18.8)$ & $17(20.0)$ & 0.85 \\
\hline Anxiety symptoms (SAST $\geq 24), n(\%)$ & $138(24.7)$ & $115(24.3)$ & $23(27.1)$ & 0.63 \\
\hline Risk of malnutrition (MUST), $\mathrm{n}(\%)$ & & & & 0.002 \\
\hline Low risk & $177(31.7)$ & $163(34.4)$ & $14(16.5)$ & \\
\hline Moderate risk & $169(30.2)$ & $142(30.0)$ & $27(31.8)$ & \\
\hline High risk & $213(38.1)$ & $169(35.7)$ & $44(51.8)$ & \\
\hline Serum albumin (g/dL) (1.5-4.9), mean \pm SD & $3.4 \pm 0.5$ & $3.3 \pm 0.5$ & $3.0 \pm 0.5$ & $<0.001$ \\
\hline \multicolumn{5}{|l|}{ In-hospital risk factors } \\
\hline ADL decline (mBI) (0-100), mean \pm SD & $3.2 \pm 8.7$ & $2.6 \pm 7.4$ & $7.0 \pm 13.2$ & 0.003 \\
\hline Length of stay $(1-30)$, mean \pm SD & $5.7 \pm 3.7$ & $5.6 \pm 3.4$ & $6.7 \pm 5.1$ & 0.055 \\
\hline
\end{tabular}

NOTE: Abbreviations: ADL, activities of daily living; APACHE II, Acute Physiology and Chronic Health Evaluation Il; mBl, modified Barthel Index; MUST, Malnutrition Universal Screening Tool; SAST, Short Anxiety Screening Test; SD, standard deviation; SPMSQ, Short Portable Mental Status Questionnaire; TZI, Tucker short Depression Rating Scale.

categorical variables and $t$ tests for continuous variables. We took a conservative approach, and used a 0.10 threshold level in univariate analysis to decide on variable inclusion in the multivariate models. To examine the at-admission readmission risk, baseline multivariate logistic regression was modeled with all pre- or at-admission variables that were associated with readmission risk in the univariate analysis. To capture the contribution of in-hospital data on readmission risk, the at-discharge multivariate logistic regression was constructed by adding in-hospital risk factors to the baseline model. To capture the odds of clinically significant functional decline that is equivalent to functional loss in $1 \mathrm{ADL}$ task, ${ }^{29}$ we divided the original $\mathrm{mBI}$ decline score by 10 . Adjusted odds ratios (OR) and $95 \%$ confidence intervals (CI) were estimated for each predictor. We used the bootstrapping technique ${ }^{30}$ (100 bootstrap subsamples) to test the ADL parameter estimates of both models. The calibration of both models was determined by the Hosmer-Lemeshow test. The discrimination of the baseline model was compared with that of the atdischarge model using the C statistic. $^{31}$ We derived the prediction score for each patient by adding the coefficients of all applicable factors from the baseline and at-discharge multivariate logistic regression models and categorized patients' risk of readmission into 5 groups: very low (0-19th centile), low (20-39th centile), medium (40-59th percentile), high (60-79th percentile), very high (80-99th percentile) and extremely high (90-99th percentile). We also examined whether when comparing the at-admission versus discharge model, new patients are identified as high risk (top $10 \%$ and $20 \%$ of risk score), as these are the patients who are targeted for intervention. Analysis was performed using IBM SPSS Statistical package version 21.0 (IBM, Armonk, NY) and Stata version 10 (StataCorp, College Station, TX).

\section{RESULTS}

Table 1 presents the baseline and in-hospital characteristics of participants with and without readmissions. The sample includes 559 community-dwelling older adults (49\% men) aged 70 to 98 years (mean age 79 years). One-third (36\%) of the participants were fully independent in ADL at admission. Eightyfive $(15.2 \%)$ patients were readmitted within 30 days of discharge. Participants who were readmitted had 
TABLE 2. Multivariate Baseline and Discharge Logistic Regression Models Predicting of 30-Day Readmission

\begin{tabular}{|c|c|c|c|c|}
\hline \multirow[b]{2}{*}{ Characteristic } & \multicolumn{2}{|c|}{ Baseline Model } & \multicolumn{2}{|c|}{ Discharge Model } \\
\hline & $\mathrm{OR}(95 \% \mathrm{Cl})$ & $P$ Value & $\mathrm{OR}(95 \% \mathrm{Cl})$ & $P$ Value \\
\hline Male & $1.57(0.89-2.77)$ & 0.12 & $1.75(0.98-3.15)$ & 0.06 \\
\hline Living alone & $1.04(0.55-1.95)$ & 0.91 & $1.06(0.56-2.01)$ & 0.86 \\
\hline Education (years) & $0.98(0.92-1.03)$ & 0.33 & $0.98(0.93-1.03)$ & 0.38 \\
\hline \multicolumn{5}{|l|}{ Chronic conditions } \\
\hline Chronic renal failure & $2.54(1.39-4.66)$ & 0.003 & $2.51(1.36-4.64)$ & 0.003 \\
\hline Malignancy & $2.45(1.38-4.32)$ & 0.002 & $2.35(1.32-4.18)$ & 0.004 \\
\hline Congestive heart failure & $1.84(1.98-3.46)$ & 0.06 & $1.83(0.97-3.46)$ & 0.06 \\
\hline Arrhythmia & $1.64(0.92-2.93)$ & 0.10 & $1.66(0.95-3.00)$ & 0.09 \\
\hline №. of medications prescribed year before index admission & $0.98(0.93-1.04)$ & 0.50 & $0.98(0.93-1.04)$ & 0.51 \\
\hline APACHE $\|$ & $0.98(0.92-1.04)$ & 0.49 & $0.97(0.91-1.04)$ & 0.36 \\
\hline No. of hospitalizations year before index admission & $1.27(1.09-1.48)$ & 0.002 & $1.26(1.08-1.46)$ & 0.004 \\
\hline \multicolumn{5}{|l|}{ Risk of malnutrition (MUST) } \\
\hline Low & Ref & & Ref & \\
\hline Moderate & $2.21(1.05-4.66)$ & 0.042 & $2.10(0.98-4.46)$ & 0.055 \\
\hline High & $3.01(1.48-6.12)$ & 0.002 & $2.88(1.41-5.91)$ & 0.004 \\
\hline Serum albumin (g/dL) & $0.41(0.24-0.69)$ & 0.001 & $0.50(0.30-0.83)$ & 0.03 \\
\hline At-admission ADL & $0.99(0.98-0.99)$ & 0.037 & $0.99(0.98-0.99)$ & 0.025 \\
\hline In-hospital ADL decline* & & & $1.32(1.02-1.72)$ & 0.034 \\
\hline Length of stay & & & $1.02(0.95-1.09)$ & 0.66 \\
\hline Model fit & C statistic $=0.81$ & & C statistic $=0.81$ & \\
\hline
\end{tabular}

NOTE: Abbreviations: ADL, activities of daily living; APACHE II, Acute Physiology and Chronic Health Evaluation II; Cl, confidence interval; MUST, Malnutrition Universal Screening Tool; OR, odds ratio. *Odds ratio for 10-unit increase in modified Barthel index.

lower at-admission ADL levels; had 1 more hospitalization in the previous year; and were more likely to have HF, CRF, arrhythmia, and malignancy, and to be at risk of malnutrition, than those who were not readmitted. Participants who were readmitted were more likely to suffer from functional decline during the index hospitalization. No significant differences were found in living arrangements or in at-admission mental and cognitive status.

Multivariate analysis (Table 2) shows that higher at-admission mBI score was associated with lower odds of readmission (OR for 1-unit increase: 0.99, 95\% CI: 0.98-0.99). Other predictors of higher readmission risk were: high or medium at-admission risk of malnutrition, malignancy, CRF, each additional hospitalization during the previous year, and lower albumin levels. Severity of illness and demographic characteristics were not significantly associated with readmission.

The at-discharge model that combined the baseline model and in-hospital risk factors showed that inhospital (from admission to discharge) ADL decline was significantly associated with readmission, as a 10point decrease in the $\mathrm{mBI}$ from admission to discharge was associated with 1.32 (95\% CI: 1.02-1.72) greater odds of readmission. LOS was not significantly associated with readmission, after controlling for baseline health status and in-hospital ADL decline. All other predictors did not markedly change from the baseline to the at-discharge model either in significance levels or in magnitude.

The discriminatory power of the baseline model was good $(C$ statistic $=0.81)$. Adding ADL decline and LOS did not change the discriminatory power of the model $(\mathrm{C}$ statistic $=0.81)$. The $P$ value of the Hosmer-Lemeshow test equaled 0.67 for the baseline model and 0.48 for the at-discharge model, indicating good calibration of both models. The $P$ values for the regression coefficients of bootstrap inference assessing the relationship between the at-admission and inhospital ADL decline odds of readmission remained stable $(P<0.05)$.

Classification of patients into risk categories by the baseline model and the discharge model (Table 3) shows that identifying patients in the top-tier category (20th highest percentile) according to information available before or at admission does not detect 6/111 $(5.4 \%)$ of patients who would have been categorized as highest-risk if information on ADL decline had been incorporated in the predictive algorithm. Additional partitioning of the top fifth group into 2 tiers (80-89th and 90-99th percentiles) shows that selection of patients in the top $10 \%$ of the baseline risk score would not have detected $7 / 55(12.7 \%)$ patients who would have been identified as high risk at discharge (data not shown).

\section{DISCUSSION}

To our knowledge, ours is the first empirical test of the simultaneous role of functioning along the hospitalization course in explaining readmission risk. ${ }^{8}$ Our results show that at-admission lower functional status and in-hospital functional decline are significant predictors of early unplanned readmission in older adults, beyond other well-known risk factors. 
TABLE 3. Classification of Patients into Risk Groups* by Baseline Characteristics (Baseline Model) and by Baseline Characteristics and Inhospital Functional Change (Discharge Model)

\begin{tabular}{cccccccc}
\hline & \multicolumn{6}{c}{ Discharge Model Risk Group } & \\
\cline { 2 - 6 } & 0 & 1 & 2 & 3 & 4 & Total No. \\
\hline Baseline model & 0 & $99(89.2)$ & $11(9.8)$ & 0 & $1(0.9)$ & 0 & 111 \\
risk group & 1 & $12(10.8)$ & $88(78.6)$ & $12(10.7)$ & 0 & 0 & 112 \\
& 2 & 0 & $13(11.6)$ & $90(80.4)$ & $8(7.1)$ & $1(0.9)$ & 112 \\
& 3 & 0 & 0 & $10(8.9)$ & $98(86.7)$ & $5(4.5)$ & 113 \\
Total no. & 4 & 0 & 0 & 0 & $6(5.3)$ & $105(94.6)$ & 111 \\
& & 111 & 112 & 112 & 113 & 111 & \\
\hline
\end{tabular}

NOTE: * $0=0$-19th percentile, $1=20-39$ th percentile, $2=40-59$ th percentile, $3=60-79$ th percentile, $4=$ 80-99th percentile

The major purpose of this study was to examine whether at-admission data can be used to detect highrisk patients for potential inclusion in readmission prevention interventions, or whether changes in ADL occurring during the index hospitalization could affect patients' risk, therefore necessitating an additional assessment at discharge. Our results show that some patients would not have been detected at admission, as their in-hospital ADL decline affects their atdischarge risk. Nonetheless, this is a small group (only $5 \%$ of patients if a targeting threshold of the highest $20 \%$ risk is used). Our findings also show that information on ADL decline during the index hospitalization does not contribute to the accuracy of readmission-risk prediction in a model that utilizes data on prior hospitalizations, baseline nutritional and functional status, and chronic morbidity (CRF and malignancy). Our results are consistent with previous studies showing the association between baseline, ${ }^{11,13,32}$ or at-admission ${ }^{13}$ functional status and readmission. However, these studies did not analyze the related contribution of in-hospital functioning to readmission risk, which was recently suggested as a feature that may significantly affect readmission risk, especially in older patients. ${ }^{8}$

Our findings are also congruent with those of a study in which LOS was not significantly associated with readmission in an elderly population. ${ }^{33}$ Our null finding can be explained by the broad set of pre- and at-admission variables, such as nutritional and functional status as well as in-hospital functional decline, included in our model, making LOS a less significant contributor than in more parsimonious models. ${ }^{28}$

Our results also show that malnutrition contributes to readmission risk beyond other well-known risk factors. Previous studies showed that malnutrition in the elderly is associated with early readmission. ${ }^{11,34}$ These studies, however, did not examine other well-known risk factors, such as previous hospitalizations, which were tested in our study, precluding identification of the contribution of malnutrition beyond other wellknown risks.
Our findings should be interpreted in light of several limitations. First, the functional, nutritional, and cognitive data were collected from participants' selfreports, which are prone to recall bias. Nonetheless, self-report is often used in large-scale studies, which preclude actual performance measurement. ${ }^{21}$ Second, our sample is of adults aged 70 years or older, and may not be representative of the 65 and older population, which is the target population for many readmission reduction interventions. ${ }^{35}$ Yet, participants were from a relatively high-functioning group of patients who were discharged to their homes, thus may resemble the over age 65 years group. Moreover, these inclusion criteria may have affected their readmission rates, which at $15 \%$ are lower than the average reported in other older adult populations. ${ }^{36}$ Nonetheless, a more heterogenic sample (in terms of baseline functional status) is needed to address the association between in-hospital functional change and readmissions as well as the discrimination of the model. Third, the attrition rate $(16 \%)$ might impact the predictive ability of the models, as patients dropped-out from the study might have had higher in-hospital deterioration. However, no significant differences between study sample and dropped-out patients in the wide range of baseline characteristics except for age and baseline albumin levels were found. Fourth, the unique characteristics of the Israeli healthcare system may affect study's generalizability. The high hospitalbed occupancy rate, stretched to the limit at $99 \%$, which is much higher than in other developed countries, ${ }^{37}$ may affect readmission rates and risk. Nonetheless, our findings may be of relevance to other populations and healthcare systems, as variables included in our model have been previously shown to affect readmission risk in other settings, ${ }^{4,6}$ and the percent of in-hospital ADL decline is similar to that reported by others. ${ }^{9}$ Future studies should examine the significance of in-hospital functioning in other older adult populations, such as greater mix of baseline functioning and myocardial infarction, HF, and COPD patients, that have been emphasized for readmission prevention by the Centers for Medicare and Medicaid Services.

\section{CONCLUSIONS}

This study shows that although both functional status and functional decline are significant predictors of readmission, in-hospital functional decline did not contribute to the discriminative ability of the model, beyond the risk factors known at admission: malnutrition, prior hospitalizations, and being previously diagnosed with CRF or malignancy. These findings call attention to the ability to predict readmission early in the index hospitalization, to enable early intervention in targeted high-risk patients. Nonetheless, further atdischarge functional assessment can detect additional patients whose readmission risk changes during the 
index hospitalization and who should be considered for inclusion in readmission reduction interventions. As suggested in previous prediction models, ${ }^{3,38}$ most of the at-admission variables examined in this study, including patient-reported measures such as functioning, are readily available in the EHR or during the atadmission intake. ${ }^{39,40}$ In settings where these assessments are not routinely performed, their implementation should be considered. These tools could be used to potentially identify patients at high risk of readmission, and accordingly, address physical function as part of routine medical care and during the acute hospitalization, and tailor adequate follow-up care after discharge. ${ }^{11}$

Disclosures: This work was supported by the Israeli Science Foundation (grant number 565/08); Clalit Health Services(grant number 04-121/ 2010); and the Israel National Institute for Health Policy Research (thesis scholarship number 35/2012). The funding agencies had no role in the design and conduct of this study, the analysis or interpretation of the data, or the preparation of the manuscript. The authors report no conflicts of interest.

\section{References}

1. Vest JR, Gamm LD, Oxford BA, Gonzalez MI, Slawson KM. Determinants of preventable readmissions in the United States: a systematic review. Implement Sci. 2010;5:1-27.

2. Garcia-Perez L, Linertova R, Lorenzo-Riera A, Vazquez-Diaz JR, Duque-Gonzalez B, Sarria-Santamera A. Risk factors for hospital readmissions in elderly patients: a systematic review. QJM. 2011;104: 639-651.

3. Shadmi E, Flaks-Manov N, Hoshen M, Goldman O, Bitterman H, Balicer RD. Predicting 30-day readmissions with preadmission electronic health record data. Med Care. 2015;53:283-289.

4. Amarasingham R, Velasco F, Xie B, et al. Electronic medical recordbased multicondition models to predict the risk of 30 day readmission or death among adult medicine patients: validation and comparison to existing models. BMC Med Inform Decis Mak. 2015;15:39.

5. Amarasingham R, Moore BJ, Tabak YP, et al. An automated model to identify heart failure patients at risk for 30-day readmission or death using electronic medical record data. Med Care. 2010;48:981-988.

6. Billings J, Blunt I, Steventon A, Georghiou T, Lewis G, Bardsley M. Development of a predictive model to identify inpatients at risk of readmission within 30 days of discharge (PARR-30). BMJ Open. 2012; 2:10.

7. Kansagara D, Chiovaro JC, Kagen D, et al. So many options, where do we start? An overview of the care transitions literature. J Hosp Med. 2016;11(3):221-230.

8. Greysen SR, Covinsky KE. Functional status-an important but overlooked variable in the readmissions equation. J Hosp Med. 2014;9: 330-331.

9. Covinsky KE, Palmer RM, Fortinsky RH, et al. Loss of independence in activities of daily living in older adults hospitalized with medical illnesses: increased vulnerability with age. J Am Geriatr Soc. 2003;51: 451-458.

10. Kwok T, Lau E, Woo J, et al. Hospital readmission among older medical patients in Hong Kong. J R Coll Physicians Lond. 1999;33:153156.

11. Greysen SR, Stijacic Cenzer I, Auerbach AD, Covinsky KE. Functional impairment and hospital readmission in Medicare seniors. JAMA Intern Med. 2015;175:559-565.

12. Hoyer EH, Needham DM, Atanelov L, Knox B, Friedman M, Brotman DJ. Association of impaired functional status at hospital discharge and subsequent rehospitalization. J Hosp Med. 2014;9:277282.

13. Laniece I, Couturier P, Drame M, et al. Incidence and main factors associated with early unplanned hospital readmission among French medical inpatients aged 75 and over admitted through emergency units. Age Ageing. 2008;37:416-422.

14. Morandi A, Bellelli G, Vasilevskis EE, et al. Predictors of rehospitalization among elderly patients admitted to a rehabilitation hospital: the role of polypharmacy, functional status, and length of stay. J Am Med Dir Assoc. 2013;14:761-767.
15. Pfeiffer E. A short portable mental status questionnaire for the assessment of organic brain deficit in elderly patients. J Am Geriatr Soc. 1975;23:433-441.

16. Zisberg A, Shadmi E, Sinoff G, Gur-Yaish N, Srulovici E, Admi H. Low mobility during hospitalization and functional decline in older adults. J Am Geriatr Soc. 2011;59:266-273.

17. Zisberg A, Shadmi E, Gur-Yaish N, Tonkikh O, Sinoff G. Hospital associated functional decline: the role of hospitalization processes beyond individual risk factors. J Am Geriatr Soc. 2015;63:55-62.

18. Flaks-Manov N, Shadmi E, Hoshen M, Balicer RD. Health information exchange systems and length of stay in readmissions to a different hospital [published online December 29, 2015]. J Hosp Med. doi: $10.1002 / \mathrm{jhm} .2535$.

19. Rennert G, Peterburg Y. Prevalence of selected chronic diseases in Israel. Isr Med Assoc J. 2001;3:404-408.

20. Knaus WA, Draper EA, Wagner DP, Zimmerman JE. APACHE II: a severity of disease classification system. Crit Care Med. 1985;13:818829.

21. Covinsky KE, Palmer RM, Counsell SR, Pine ZM, Walter LC, Chren MM. Functional status before hospitalization in acutely ill older adults: validity and clinical importance of retrospective reports. $J \mathrm{Am}$ Geriatr Soc. 2000;48:164-169.

22. Shah S, Vanclay F, Cooper B. Improving the sensitivity of the Barthel index for stroke rehabilitation. J Clin Epidemiol. 1989;42:703-709.

23. Tucker M, Ogle S, Davison J, Eilenberg M. Validation of a brief screening test for depression in the elderly. Age Ageing. 1987;16:139144.

24. Sinoff G, Ore L, Zlotogorsky D, Tamir A. Short Anxiety Screening Test - a brief instrument for detecting anxiety in the elderly. Int J Geriatr Psychiatry. 1999;14:1062-1071.

25. Sinoff G, Ore L, Zlotogorsky D, Tamir A. Does the presence of anxiety affect the validity of a screening test for depression in the elderly? Int J Geriatr Psychiatry. 2002;17:309-314.

26. Elia M. The 'MUST' report. Nutritional screening of adults: a multidisciplinary responsibility (executive summary). Available at: http:// www.bapen.org.uk/pdfs/must/must_exec_sum.pdf. Accessed July 10, 2015.

27. Somes GW, Kritchevsky SB, Shorr RI, Pahor M, Applegate WB. Body mass index, weight change, and death in older adults: the systolic hypertension in the elderly program. Am J Epidemiol. 2002;156:132138.

28. van Walraven C, Dhalla I, Bell C, Etchells E, et al. Derivation and validation of an index to predict early death or unplanned readmission after discharge from hospital to the community. CMAJ. 2010;182: 551-557.

29. Buurman BM, van Munster BC, Korevaar JC, de Haan RJ, de Rooij SE. Variability in measuring (instrumental) activities of daily living functioning and functional decline in hospitalized older medical patients: a systematic review. J Clin Epidemiol. 2011;64:619-627.

30. Steyerberg EW, Harrell FE Jr, Borsboom GJ, Eijkemans MJ, Vergouwe Y, Habbema JD. Internal validation of predictive models: efficiency of some procedures for logistic regression analysis. J Clin Epidemiol. 2001;54:774-781.

31. Kansagara D, Englander H, Salanitro A, et al. Risk prediction models for hospital readmission: a systematic review. JAMA. 2011;306: $1688-1698$.

32. Soley-Bori M, Soria-Saucedo R, Ryan CM, et al. Functional status and hospital readmissions using the medical expenditure panel survey. J Gen Intern Med. 2015;30:965-972.

33. Cotter PE, Bhalla VK, Wallis SJ, Biram RW. Predicting readmissions: poor performance of the LACE index in an older UK population. Age Ageing. 2012;41:784-789.

34. Silverstein MD, Qin H, Mercer SQ, Fong J, Haydar Z. Risk factors for 30-day hospital readmission in patients $\geq 65$ years of age. Proc (Bayl Univ Med Cent). 2008;21:363-372.

35. Center for Outcomes Research \& Evaluation. 2014 measure updates and specifications report: hospital-wide all-cause unplanned readmission-version 3.0. Available at: https://www.qualitynet.org. Published July 2014. Accessed March 14, 2016.

36. Jencks SF, Williams MV, Coleman EA. Rehospitalizations among patients in the Medicare fee-for-service program. N Engl J Med. 2009; 360:1418-1428.

37. Meydan C, Haklai Z, Gordon B, Mendlovic J, Afek A. Managing the increasing shortage of acute care hospital beds in Israel. J Eval Clin Pract. 2015;21:79-84.

38. Billings J, Dixon J, Mijanovich T, Wennberg D. Case finding for patients at risk of readmission to hospital: development of algorithm to identify high risk patients. BMJ. 2006;333:327.

39. Rasmussen $\mathrm{HH}$, Holst M, Kondrup J. Measuring nutritional risk in hospitals. Clin Epidemiol. 2010;2:209-216.

40. Hinami K, Smith J, Deamant CD, DuBeshter K, Trick WE. When do patient-reported outcome measures inform readmission risk? J Hosp Med. 2015;10:294-300. 